\title{
Learning from nature: Fabrication of colloidal PCs with controllable wettability
}

Colloidal photonic crystals (PCs) with desired wettabilities are of particular importance in various applications. Inspired by biological PCs, such as those of butterfly wings and peacock feathers, which all have evolved to have specific wettability, Professor Song Yanlin and his group in the Laboratory of New Materials, Institute of Chemistry, Chinese Academy of Sciences developed unique approaches to engineer colloidal PCs with desired wettability, based on the well-designed building blocks of latex particles. The range of wettability of these colloidal PC films could be tailored from superhydrophobicity to superhydrophilicity and even superoleophobicity, by varying the surface chemical composition and surface morphology of the latex particles. Their work, entitled "Bioinspired colloidal photonic crystals with controllable wettability", was published in Acc Chem Res, 2011, 44(6): 405-415.

Colloidal PCs, using the periodic arrangement of latex particles, have attracted much interest due to their unique capability to manipulate the propagation of light and their consequent potential for use in novel optical devices. Colloidal PCs exhibiting structural colors because of the match of the periodicity with the wavelength of visible light have already found broad applications. Colloidal PCs with special wettabilities can endow these materials with novel functionalities such as self-cleaning properties for optical devices, full wetting and spreading of precursors to facilitate the complete infiltration into an opal template, the full diffusion of a catalyst into an opal reaction system, and the rapid detection of liquid in microfluidic systems.

In nature, many biological PCs have specific wettabilities in addition to the well-known iridescent structural color. Iridescence is used for species recognition and mate selection by insects, birds, fish, and reptiles. Specific wettability is necessary for the survival of creatures in nature. For instance, the superhydrophobicity of butterfly wings and peacock feathers shields them from rainfall as rain drops cannot stay on those surfaces. At the same time, dust particles on these surfaces can be easily removed. The superhydrophobicity of a mosquito's compound eyes favors it with good vision in a humid environment because fog droplets easily roll off the surface. Moreover, longhorn beetles show us an ingenious example of the harmony of PCs and special wettability. An amazing camouflage behavior has been observed as the color pattern on their hydrophilic elytra changes from yellow to red when environmental humidity increases and vice versa - the color can return to red when the humidity drops.

Inspired by these unique properties of biological PCs, colloidal PCs with controllable wettability are based mainly on two approaches: either coating materials with specific wettability onto the surface of colloidal crystal films or modifying the surface properties of latex particles. For example, the wettability of as-prepared colloidal crystals can be controlled from superhydrophobic to superhydrophilic by varying the assembly temperature, assembly $\mathrm{pH}$, light irradiation or different electronic positions, depending on the well-designed latex surface or suitable infiltration of the functional materials into an opal template. As-prepared colloidal PCs show important applications in visible humidity sensing, highly efficient catalysis, amongst others. The fabrication of colloidal PCs with controllable wettability will greatly broaden the applications of PCs in various optical devices.

The research on colloidal PCs with special wettability is still on-going and numerous challenges remain. For example, the design and fabrication of latex spheres with novel morphology and structure (such as mushroom-cap shaped, snowman, or other anisotropic latex structures) will greatly influence their wettability characteristics. It is also desired to design, synthesize, and apply new materials to PCs that can respond to exterior stimuli. Exploring new PCs in nature and establishing the relationships between their structures and functions will provide effective ways to prepare novel functional materials and learning from nature in depth will be of great importance for the development of artificial materials..

Based on well-designed latex particles, including their surface morphologies, surface chemical composition, and particle dimensions, colloidal PCs with controllable wettability and iridescent structural colors may be obtained. This is expected to be an effective route to the bioinspired design of colloidal PCs with novel functionalities.

See the article: Wang J X, Zhang Y Z, Wang S T, et al. Bioinspired colloidal photonic crystals with controllable wettability. Acc Chem Res, 2011, 44: $405-415$

Open Access This article is distributed under the terms of the Creative Commons Attribution License which permits any use, distribution, and reproduction in any medium, provided the original author(s) and source are credited. 